\title{
An Integrability Condition for Simple Lie Groups II
}

Maung MIN-OO

Department of Mathematics \& Statistics, McMaster University, Hamilton, Canada

E-mail:minoo@mcmaster.ca

Received December 17, 2014, in final form March 26, 2015; Published online April 01, 2015

http://dx.doi.org/10.3842/SIGMA.2015.027

\begin{abstract}
It is shown that a simple Lie group $G\left(\neq \mathrm{SL}_{2}\right)$ can be locally characterised by an integrability condition on an $\operatorname{Aut}(\mathfrak{g})$ structure on the tangent bundle, where $\operatorname{Aut}(\mathfrak{g})$ is the automorphism group of the Lie algebra of $G$. The integrability condition is the vanishing of a torsion tensor of type $(1,2)$. This is a slight improvement of an earlier result proved in [Min-Oo M., Ruh E.A., in Differential Geometry and Complex Analysis, Springer, Berlin, 1985, 205-211].
\end{abstract}

Key words: simple Lie groups and algebras; $G$-structure

2010 Mathematics Subject Classification: 53C10; 53C30

\section{Introduction}

This is a very short addendum to a paper that I wrote with E.A. Ruh in 1985 [3], where we characterized a simple Lie group $G\left(\neq \mathrm{SL}_{2}\right)$ by an integrability condition on an $\operatorname{Aut}(\mathfrak{g})$ structure on the tangent bundle, where Aut( $(\mathfrak{g})$ is the automorphism group of the Lie algebra of $G$. The condition was on a tensor of type $(1,3)$. In this paper we derive a slightly improved version of that result by using a more natural integrability condition involving a tensor of type $(1,2)$, namely the "canonical" torsion for the given structure.

The classical theorems of Sophus Lie characterise what we now call Lie groups (locally) by their Lie algebras, which are vector fields whose Lie brackets are "constant" (structure constants). Nowadays, geometric structures are usually described by infinitesimal tensorial objects such as a metric or an almost-complex structure defining what is known as a $G$-structure (a reduction of the frame bundle to a subgroup). Our result gives a characterisation of a simple (local) Lie group $\neq \mathrm{SL}_{2}$ ) by the torsion of the appropriate $G$-structure, namely the automorphism group of its Lie algebra, which in this case is the same as $G$, up to coverings and connected components. Our result is different from the classical theorems because it does not hold for a general Lie group, only for simple Lie groups of rank $\geq 2$. The main reason is that we use the deep result about holonomy groups by Berger [1] and Simons [5]. More precisely, we need the purely algebraic version proved by Simons [5] about the rigidity of holonomy groups of rank $\geq 2$, acting non-transitively on the unit sphere (that is the reason why we have to exclude $\mathrm{SL}_{2}$, which is of rank one). One can find an independent simple algebraic proof of Simon's result for simple Lie groups of rank $\geq 2$ in our earlier paper [3].

\section{The result}

Let $M$ an $n$-dimensional manifold and $\mathfrak{g}$ a Lie algebra of the same dimension. An Aut( $\mathfrak{g})$ structure, in the sense of $G$-structures is a reduction of the frame bundle of $M$ to the subgroup $\operatorname{Aut}(\mathfrak{g}) \subset \operatorname{GL}(n ; \mathbb{R})$. Such a structure is determined by a skew-symmetric tensor $T: T M \otimes$ $T M \longrightarrow T M$ of type $(1,2)$ satisfying the Jacobi identity:

$$
T(X, Y)+T(Y, X)=0,
$$




$$
T(X, T(Y, Z))+T(Y, T(Z, X))+T(Z, T(X, Y))=0 .
$$

$T$ defines a Lie algebra structure for the tangent space at each point.

The $\operatorname{Aut}(\mathfrak{g})$-structure is given by all frames $u: \mathfrak{g} \cong T_{p} M$ satisfying

$$
u([A, B])=T(u(A), u(B))
$$

and $G$ acts through inner automorphisms: $u g=u \circ \mathrm{ad}_{g}$. If $u$ is a moving frame (a local section of the principal bundle), then we do not assume that the Lie bracket of vector fields $[u(A), u(B)]$ is the same as $u([A, B])$ (otherwise we will be just reproving Lie's original theorems).

For a semi-simple Lie algebra $\mathfrak{g}$, the first Lie algebra cohomology group $H^{1}(\mathfrak{g}$; ad) with values in the adjoint representation vanishes and so almost all automorphisms are inner automorphisms, in the sense that the inner automorphisms form a normal subgroup of finite index. In fact, $H^{2}(\mathfrak{g}$; ad) also vanishes (see [2]) and this is crucial for our proof. A semi-simple real Lie algebra $\mathfrak{g}$ has a natural bi-invariant non-degenerate inner product (not necessarily positive-definite) given by the Killing form $\langle X, Y\rangle=-B_{\mathfrak{g}}(X, Y)$. This defines a (pseudo-)Riemannian metric on $M$, which we shall again denote by $\langle$,$\rangle . Let D$ denote the Levi-Civita connection of this metric. In [3], we looked at the following tensor $d^{D} T$ of type $(1,3)$

$$
d^{D} T(X, Y, Z)=\left(D_{X} T\right)(Y, Z)+\left(D_{Y} T\right)(Z, X)+\left(D_{Z} T\right)(X, Y)
$$

and we proved the following result

Theorem 2.1 ([3]). Let $\mathfrak{g}$ be a semi-simple real Lie algebra different from $\mathfrak{s l}_{2}(\mathbb{C})$ or any of its real forms. Then $d^{D} T=0$ iff $(M,\langle\rangle$,$) is either locally isometric to a Lie group G$ (with Lie algebra $\mathfrak{g}$ ) with its unique (up to a constant factor) bi-invariant metric, or else is flat.

The purpose of this paper is to introduce a more natural integrability condition. First we need to define "dual" bases $\left\{e_{k}\right\}$ and $\left\{e^{j}\right\}$ for $\mathfrak{g}$ satisfying $\left\langle e_{k}, e^{j}\right\rangle=\delta_{k}^{j}$ (this is standard procedure in this theory):

Case 1. If $\mathfrak{g}$ is compact, then the metric $\langle$,$\rangle is positive definite and we just set e_{k}=e^{k}$, where $\left\{e_{k}\right\}$ is an orthonormal base for the metric.

Case 2. If $\mathfrak{g}$ is not compact, we split $\mathfrak{g}=\mathfrak{k} \oplus \mathfrak{m}$ where the metric $\langle$,$\rangle is positive-definite on \mathfrak{k}$ (the maximal compact subalgebra) and negative-definite on the vector space $\mathfrak{m}$. On $\mathfrak{k}$ we define $e_{k}=e^{k}$, where $\left\{e_{k}\right\}$ is an orthonormal base. On $\mathfrak{m}$, we set $e^{j}=-e_{j}$.

These define (local) frames for the $\operatorname{Aut}(\mathfrak{g})$-structure on $M$.

We now define our integrability tensor $\tau$ as follows

$$
\tau(X, Y)=\sum_{k}\left(T\left(e_{k},\left(D_{X} T\right)\left(Y, e^{k}\right)\right)-T\left(e_{k},\left(D_{Y} T\right)\left(X, e^{k}\right)\right)\right) .
$$

This might look a bit complicated at first sight, but it is a tensor of type $(1,2)$, so it is more in line with many other integrability conditions. It is clear that $\tau$ is a well defined tensor on $M$ (independent of the choice of the dual base), given the Aut $(\mathfrak{g})$-structure. The main reason why $\tau$ is the correct tensor is because it is the torsion of the following connection:

\section{Definition 2.2.}

$$
\nabla_{X} Y=D_{X} Y+A_{X} Y
$$

where

$$
A_{X} Y=\sum_{k} T\left(e_{k},\left(D_{X} T\right)\left(Y, e^{k}\right)\right) .
$$


Now here is the key fact about this connection:

Proposition 2.3. $T$ is parallel with respect to the connection $\nabla$, i.e.,

$$
\nabla T=0 .
$$

Since the (pseudo-)Riemannian metric $\langle$,$\rangle on M$ is defined algebraically using only the Killing form of the Lie algebra structure on each tangent space $T_{p} M$, given by the tensor $T$, we have the following corollaries:

Corollary 2.4. $\nabla$ is a metric connection with torsion $\tau$ :

$$
\left\langle A_{X} Y, Z\right\rangle+\left\langle A_{X} Z, Y\right\rangle=0
$$

\section{Corollary 2.5.}

$$
\tau=0 \Leftrightarrow A=0 \quad \Leftrightarrow \quad D T=0 \quad \Leftrightarrow \quad d^{D} T=0 .
$$

Remark 2.6. In the language of [6] or [4], $\tau$ is called the torsion of the Aut( $\mathfrak{g})$-structure, which is an invariant that depends only on the $\operatorname{Aut}(\mathfrak{g})$-structure and $\nabla$ is the explicit connection that realises this torsion.

So in view of Theorem 2.1, proved in the earlier paper [3], Proposition 2.3 now implies the main result of this short note:

Theorem 2.7. Let $\mathfrak{g}$ be a semi-simple real Lie algebra different from $\mathfrak{s l}_{2}(\mathbb{C})$ or any of its real forms. Then $\tau=0$ iff $(M,\langle\rangle$,$) is either locally isometric to a Lie group G$ (with Lie algebra $\mathfrak{g}$ ) with its unique (up to a constant factor) bi-invariant metric, or else is flat.

Proof of Proposition 2.3. Let $v \in T_{p} M$, and let us denote for simplicity the the covariant derivative $D_{v} T$ by $T^{\prime}$. Then by differentiating the Jacobi identity (2.1), we obtain

$$
\begin{aligned}
& T\left(T^{\prime}(X, Y), Z\right)+T\left(T^{\prime}(Y, Z), X\right)+T\left(T^{\prime}(Z, X), Y\right) \\
& \quad+T^{\prime}(T(X, Y), Z)+T^{\prime}(T(Y, Z), X)+T^{\prime}(T(Z, X), Y)=0 .
\end{aligned}
$$

In the language Lie algebra cohomology, this is simply a cocycle condition on $T^{\prime}$ can simply be expresses as

$$
d_{\mathrm{ad}} T^{\prime}=0,
$$

where $d_{\text {ad }}$ is the co-boundary operator for two-forms with values in the adjoint representation. It is well known [2] that $H^{2}(\mathfrak{g} ; \mathrm{ad}) \cong 0$. Hence

$$
d_{\mathrm{ad}} T^{\prime}=0 \Rightarrow T^{\prime}=d_{\mathrm{ad}} A
$$

for some $A$, where $A$ is a $\mathfrak{g}$-valued one-form. i.e. a "gauge transformation" $A: T_{p} M \rightarrow \mathfrak{g} \cong T_{p} M$. In fact it can be easily checked that $A$ can be explicitly written as (see [2, p. 90]):

$$
A(X)=\sum_{k} T\left(e_{k}, T^{\prime}\left(X, e^{k}\right)\right) .
$$

This is in fact the "optimal" choice of $A$ given the metric structure, in the sense of Hodge theory, since $A$ satisfies $d_{\mathrm{ad}}^{\star} A=0$, where $d_{\mathrm{ad}}^{\star}$ is the adjoint of $d_{\mathrm{ad}}$ with respect to the natural bi-invariant metric. We have then

$$
T^{\prime}(X, Y)=\left(d_{\mathrm{ad}} A\right)(X, Y)=T(A X, Y)+T(X, A Y)-A(T(X, Y)),
$$

SO

$$
\begin{aligned}
\left(\nabla_{v} T\right)(X, Y) & =\left(D_{v} T\right)(X, Y)+A_{v}(T(X, Y))-T\left(A_{v} X, Y\right)-T\left(X, A_{v} Y\right) \\
& =T^{\prime}(X, Y)-d_{\mathrm{ad}} A(X, Y)=0 .
\end{aligned}
$$




\section{References}

[1] Berger M., Sur les groupes d'holonomie homogène des variétés à connexion affine et des variétés riemanniennes, Bull. Soc. Math. France 83 (1955), 279-330.

[2] Jacobson N., Lie algebras, Interscience Tracts in Pure and Applied Mathematics, Vol. 10, Interscience Publishers, New York - London, 1962.

[3] Min-Oo M., Ruh E.A., An integrability condition for simple Lie groups, in Differential Geometry and Complex Analysis, Springer, Berlin, 1985, 205-211.

[4] Min-Oo M., Almost symmetric spaces, Astérisque 163-164 (1988), 221-246.

[5] Simons J., On the transitivity of holonomy systems, Ann. of Math. 76 (1962), 213-234.

[6] Sternberg S., Lectures on differential geometry, 2nd ed., Chelsea Publishing Co., New York, 1983. 